\title{
Antibiotic therapy in acute gastroenteritis: a single-center retrospective cohort study
}

\author{
Maher Homsi ${ }^{a}$, Bhanu Singh ${ }^{\mathrm{b}}$, Muaataz Azawi ${ }^{\mathrm{b}}$, Ankur Panchall ${ }^{\mathrm{b}}$, Nabeeh Hauter ${ }^{\mathrm{b}}$, Carolyn Salafiac, \\ Joshua Aron ${ }^{d}$
}

University of Florida College of Medicine, Gainesville, Florida; Icahn School of Medicine at Mount Sinai, NY; The Mount Sinai School of Medicine, NY, USA

\begin{abstract}
Background Acute gastroenteritis (AGE) is a common reason for emergency department visits and hospitalizations. The role of antibiotics in AGE is unclear, as the current literature shows only a minor impact on the duration of symptoms and the overall clinical course. Our goal was to assess whether antibiotic therapy in patients with AGE affects the length of hospital stay (LOS).

Methods In a retrospective study, we evaluated 479 patients admitted to the hospital with a diagnosis of AGE. The study compared the 219 patients (46\%) treated with antibiotics to the remainder treated with supportive therapy. The diagnosis of AGE was made either clinically or based on imaging findings. The primary outcome of this study was to compare the LOS in days between both groups.

Results Patients treated with antibiotics had a similar LOS to those treated with supportive therapy ( 2.62 vs. 2.66 days, $\mathrm{P}=0.77$ ). Patients with presumed sepsis had a higher likelihood of receiving antibiotics compared to those without presumed sepsis (risk ratio $1.49,62.5 \%$ vs. $41.95 \% ; \mathrm{P}<0.001$ ). In this subgroup, patients who received antibiotics had a slightly shorter LOS than those who received only supportive therapy, but the difference was not statistically significant ( 2.09 vs. 2.54 days, $\mathrm{P}=0.69$ ).

Conclusion We found no difference in the LOS for hospitalized patients with AGE treated with antibiotics when compared to supportive therapy. This calls into question the role of antibiotics in the management of AGE.
\end{abstract}

Keywords Acute gastroenteritis, antibiotic therapy, length of hospital stay, diarrheal disease

Ann Gastroenterol 2019; 32 (6): 1-5

\section{Introduction}

Acute gastroenteritis (AGE) presents a major burden to the global and the United States healthcare systems, as it is one of the most common diagnoses for emergency department

Department of a Internal Medicine, Division of Hospital Medicine, University of Florida College of Medicine, Gainesville, Florida (Maher Homsi); ' Internal Medicine, Icahn School of Medicine at Mount Sinai (Elmhurst Hospital Center), New York (Bhanu Singh, Muaataz Azawi, Ankur Panchal, Nabeeh Hauter); ' Internal Medicine, The Mount Sinai School of Medicine, New York (Carolyn Salafia); ${ }^{\mathrm{I}}$ Internal Medicine, Division of Gastroenterology, Icahn School of Medicine at Mount Sinai (Elmhurst Hospital Center), New York (Joshua Aron)

Conflict of Interest: None

Correspondence to: Maher Homsi, MD, Assistant Professor of Medicine, University of Florida, Gainesville, FL, USA, e-mail: mhomsi@ufl.edu

Received 11 July 2019; accepted 23 September 2019; published online 9 October 2019

DOI: https://doi.org/10.20524/aog.2019.0422 visits and hospitalizations [1-3]. According to the Centers for Disease Control and Prevention, it is estimated that there are 37.2 million cases of infectious gastroenteritis annually in the United States alone, with 228,744 hospitalizations and 2,612 deaths [4]. The Healthcare Cost and Utilization Project (HCUP-US) found the average length of hospital stay (LOS) to be 2.9 days nationwide, with an average cost of $\$ 11,910$ per stay [5]. While there are a broad variety of pathogens responsible for acute infectious gastroenteritis, the majority of cases are attributed to viral illness [4-8]. The management of patients presenting with AGE is primarily supportive and consists of rehydration [9-12]. Oral hydration is the preferred modality for fluid repletion [13-15]. However, in patients with severe hypovolemia or an inability to tolerate oral hydration (including impaired mental status or severe ileus), intravenous hydration is a recommended alternative [13-15]. The role of antibiotic therapy in the management of AGE is unclear. There are limited data in the literature to strongly support their use. To our knowledge, no prior studies had looked at the role of antibiotic therapy in hospitalized patients with AGE and the LOS. 


\section{Patients and methods}

\section{Study design}

A single-center retrospective cohort study identified 498 adult patients who had an emergency department visit and required hospitalization for AGE between January 2005 and July 2015. Of these, 19 patients did not meet the inclusion criteria (see below) and were removed from the study, leaving a total of 479 patients evaluated (Fig. 1). We identified patients with AGE based on the clinical diagnosis made by the treating physician. The diagnosis was based either on the clinical presentation alone, or on the clinical presentation and imaging findings from a computed tomography scan of the abdomen, if applicable. Inclusion criteria included adult patients with diagnosis of AGE as above, older than 18 years, no history of inflammatory bowel disease, no active diagnosis of diverticulitis, active Clostridium difficile (C. difficile) infection, or concurrent infection (e.g., pneumonia or urinary tract) during the same admission. The primary outcome of this study was to compare the LOS in days between patients who received antibiotic therapy and patients who received supportive therapy alone. A subanalysis was performed to eliminate a potential confounder in our study, patients with a prolonged hospital stay ( $>10$ days) were excluded from the study and the LOS was re-measured.

QuadraMed Computerized Patient Record software was accessed for extraction of patient age, sex, body mass index (BMI), LOS, presenting symptoms such as nausea, vomiting, diarrhea, bloody diarrhea or abdominal pain, initial vital signs including body temperature, heart rate and systolic blood pressure, laboratory tests including white cell count and lactate level, stool culture, stool leukocytes and C. difficile testing, and the antibiotic therapy administration during hospitalization. A diagnosis of presumed sepsis of enteric source was made if 2 or more of the criteria for systemic inflammatory response syndrome (SIRS) were present: tachycardia (pulse $>90$ beats/min), hypotension (systolic blood pressure $<90 \mathrm{mmHg}$ ), leukocytosis (white blood cell count $>12,000)$, fever $\left(>38.0^{\circ} \mathrm{C}\right)$ and increased lactate levels (when determined). This study was approved by the Institutional Review Board of the Mount Sinai School of Medicine.

\section{Statistical analysis}

IBM SPSS for Windows (version 24; IBM SPSS Inc.) was used for the statistical analysis. Categorical variables were compared using contingency tables or chi-square test. Continuous variables were checked for normality and those found to be normally distributed (e.g., age and BMI) were compared to our categorical predictor variables using ANOVA. LOS was not normally distributed; therefore, its relationships with other variables were compared using the Mann-Whitney $U$ test. A P-value less than 0.05 was considered significant.

\section{Results}

The average age of the patients in our study was $54 \pm 18$ years and most were women (57\%). Abdominal pain was the most common presenting symptom (77.8\%), followed by nausea, diarrhea, vomiting, and bloody diarrhea, respectively $(72.2 \%$, $69.7 \%, 65.3 \%, 9.8 \%$ ). Leukocytosis was found in $36.1 \%$, hypotension in $18.1 \%$, and fever was only documented in $10.6 \%$ of our total population (Fig. 2). A fecal leukocyte test was performed in 187 patients (39\%), of whom 30 had positive results (16\%). Stool cultures were ordered in 199 patients (42\%); only 15 patients had positive stool cultures (7.5\%).

Having any of the following symptoms or signs-abdominal pain, diarrhea, bloody diarrhea, fever, leukocytosis, hypotension or tachycardia-was associated with a higher likelihood of antibiotic administration $(\mathrm{P}<0.008,<0.001,<0.001,<0.001$, $<0.001,=0.05,=0.04$, respectively). Patients who complained

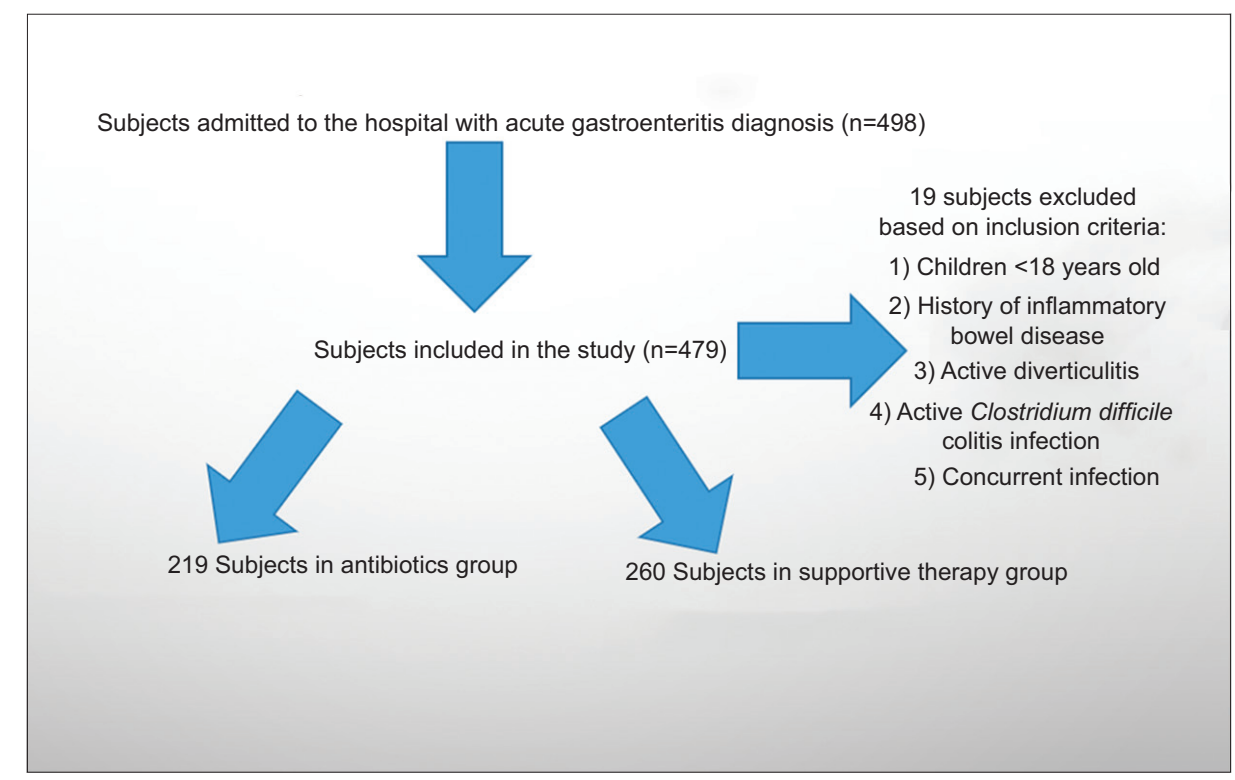

Figure 1 Study design with exclusion criteria 


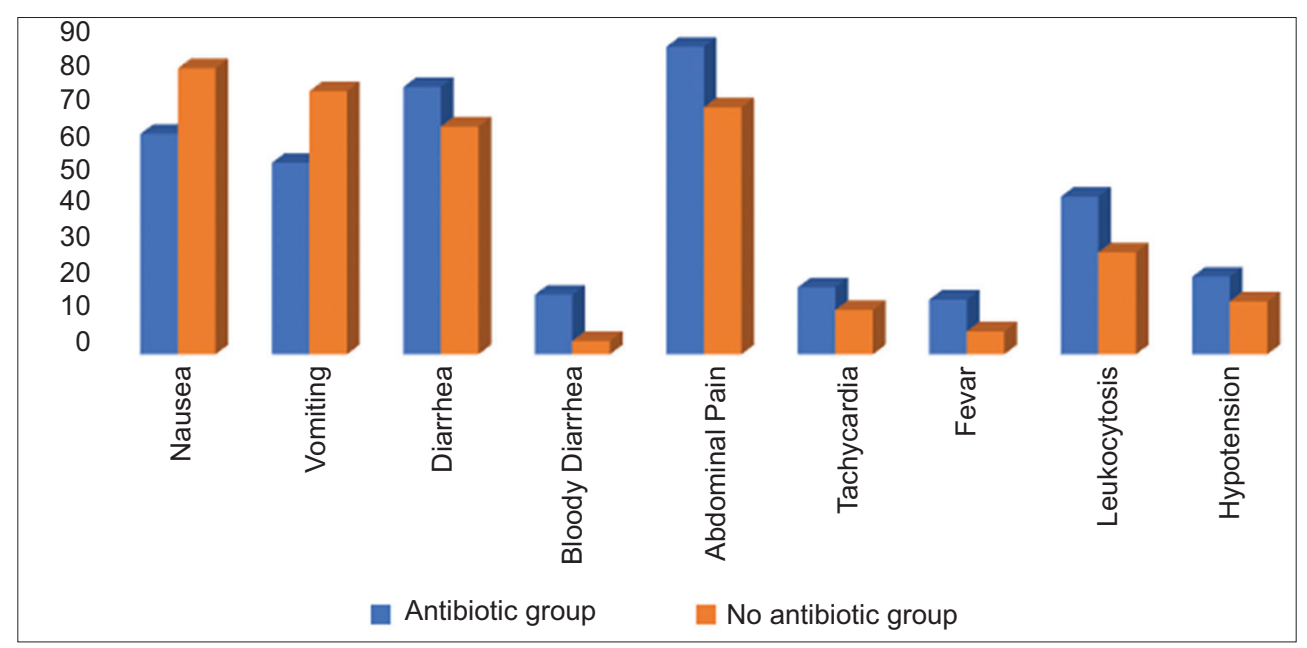

Figure 2 The percentage of patients with each symptom and sign

Table 1 Baseline characteristics of patients in the antibiotic therapy group and the supportive therapy group

\begin{tabular}{|c|c|c|c|c|}
\hline Characteristics & $\begin{array}{l}\text { All study population } \\
\qquad(\mathrm{N}=479)\end{array}$ & $\begin{array}{l}\text { Antibiotic therapy } \\
\text { group }(\mathrm{N}=219)\end{array}$ & $\begin{array}{l}\text { Supportive therapy } \\
\text { group }(\mathrm{N}=260)\end{array}$ & P-value \\
\hline Age (SD) & $54(18)$ & $54 \pm 19$ & $55 \pm 18$ & 0.52 \\
\hline Male: Female & $206: 273$ & 91:128 (59\%:41\%) & 115:145 (58\%:42\%) & 0.55 \\
\hline BMI (SD) & $26.4(5.9)$ & $26.6 \pm 5.6$ & $26.5 \pm 5.6$ & 0.89 \\
\hline Length of stay (days) (range) & $2.65(1-56)$ & $2.62(1-56)$ & $2.66(1-20)$ & 0.77 \\
\hline Nausea & $346(72.2 \%)$ & $136(62.5 \%)$ & $210(81.1 \%)$ & $<0.001$ \\
\hline Vomiting & $313(65.3 \%)$ & $119(54.3 \%)$ & $194(74.6 \%)$ & $<0.001$ \\
\hline Diarrhea & $334(69.7 \%)$ & $166(75.8 \%)$ & $168(64.6 \%)$ & $<0.008$ \\
\hline Bloody diarrhea & $47(9.81 \%)$ & $37(16.9 \%)$ & $10(3.8 \%)$ & $<0.001$ \\
\hline Abdominal pain & $373(77.8 \%)$ & $191(87.2 \%)$ & $182(70 \%)$ & $<0.001$ \\
\hline Leukocytosis $\geq 12,000$ & $173(36.1 \%)$ & $98(44.7 \%)$ & $75(28.9 \%)$ & $<0.001$ \\
\hline Fever $\geq 38^{\circ} \mathrm{C}$ & $51(10.6 \%)$ & $34(15.5 \%)$ & $17(6.5 \%)$ & $<0.001$ \\
\hline Hypotension $<90 \mathrm{mmHg}$ & $87(18.1 \%)$ & $48(22 \%)$ & $39(15 \%)$ & 0.05 \\
\hline Tachycardia $>90 / \mathrm{min}$ & $75(15.6 \%)$ & $42(19 \%)$ & $33(12.6 \%)$ & $<0.04$ \\
\hline Presumed sepsis & $88(18.4 \%)$ & $55(27.3 \%)$ & $33(12.7 \%)$ & $<0.001$ \\
\hline Positive fecal leukocyte test & $30(16 \%)$ & & & \\
\hline Positive stool cultures & $15(7.5 \%)$ & & & \\
\hline Antibiotic administration & $219(45.7 \%)$ & & & \\
\hline
\end{tabular}

BMI, body mass index; $S D$, standard deviation

of nausea and/or vomiting were less likely to receive antibiotic therapy $(\mathrm{P}<0.001, \mathrm{P}<0.001$, respectively).

Our 2 groups comprised, first, all patients with AGE treated with antibiotic therapy $(\mathrm{N}=219,46 \%)$, and second, with AGE diagnosis treated with supportive therapy alone $(\mathrm{N}=260,54 \%)$. Patients who had received antibiotic therapy had a similar LOS to those treated with supportive therapy and intravenous fluids alone (2.62 days [range 1-56], vs. 2.66 days [range 1-20], $\mathrm{P}=0.77, \mathrm{n}=479$ ) (Table 1). When adjustment was made for a small number of patients with a hospital stay longer than 10 nights, there was still no impact of antibiotic therapy on LOS ( 2.33 days vs. $2.33, \mathrm{P}=0.86, \mathrm{n}=467$ ).
Eighty-eight (18\%) of our patients had severe disease and presumed sepsis of enteric source. Of those patients, $55(62.5 \%)$ had received antibiotic therapy. The LOS of these very ill patients was slightly but not statistically significantly shorter compared to those who did not receive antibiotic therapy (2.09 days vs. 2.54 days, $\mathrm{P}=0.69$ ). Diarrhea was the most common presenting symptom in $88 \%$ of cases, followed by abdominal pain in $77 \%$. Patients with presumed sepsis were more likely to receive antibiotic therapy compared to those without presumed sepsis (risk ratio $1.49,62.5 \%$ vs. $41.95 \%$; $\mathrm{P}<0.001$ ) (Table 2). 
Table 2 Baseline characteristics of patients in the antibiotic therapy group and the supportive therapy group who had presumed sepsis with a suspected enteric source

\begin{tabular}{lccc}
\hline Characteristics & $\begin{array}{c}\text { Antibiotic } \\
\text { therapy } \\
\text { group (N=55) }\end{array}$ & $\begin{array}{c}\text { Supportive } \\
\text { therapy } \\
\text { group (N=33) }\end{array}$ & P-value \\
\hline Age (SD) & $55 \pm 20.4$ & $56 \pm 17$ & 0.96 \\
\hline M: F & $\begin{array}{c}20: 35 \\
(36 \%: 64 \%)\end{array}$ & $\begin{array}{c}14: 19 \\
(42 \%: 58 \%)\end{array}$ & 0.57 \\
\hline BMI (SD) & $27 \pm 7$ & $28 \pm 7$ & 0.59 \\
\hline $\begin{array}{l}\text { Length of stay } \\
\text { (days) (range) }\end{array}$ & $2.09(1-5)$ & $2.54(1-10)$ & 0.69 \\
\hline Nausea & $36(65.5 \%)$ & $25(75.8 \%)$ & 0.22 \\
\hline Vomiting & $30(54.5 \%)$ & $20(60.6 \%)$ & 0.37 \\
\hline Diarrhea & $47(85.5 \%)$ & $30(90.9 \%)$ & 0.34 \\
\hline Bloody diarrhea & $13(23.6 \%)$ & $1(3 \%)$ & 0.008 \\
\hline Abdominal pain & $45(81.8 \%)$ & $23(69.7 \%)$ & 0.14 \\
\hline Leukocytosis $\geq 12.000$ & $42(76.4 \%)$ & $24(72.7 \%)$ & 0.44 \\
\hline Fever $\geq 100.4\left(38^{\circ} \mathrm{C}\right)$ & $27(49.1 \%)$ & $12(36.4 \%)$ & 0.17 \\
\hline Hypotension $<90$ & $29(52.7 \%)$ & $17(51.5 \%)$ & 0.54 \\
\hline Tachycardia $>90$ & $31(56.4 \%)$ & $17(51.5 \%)$ & 0.41 \\
\hline${ }^{*}$ BMI body $m a s s i n d e x: 5 D, 5 t a n d a r d$ deviation & & \\
\hline
\end{tabular}

${ }^{{ }^{*} B M I}$, body mass index; $S D$, standard deviation

\section{Discussion}

The average LOS of hospitalized patients with AGE in our study was 2.65 days, similar to data from HCUP-US, which found the average LOS to be 2.9 days nationwide [25]. In our study, $46 \%$ of patients presenting with symptoms consistent with AGE were given antibiotic therapy; $79 \%$ of patients presenting with bloody diarrhea had received antibiotic therapy and around $63 \%$ of patients with presumed sepsis of enteric source had received antibiotic therapy. Interestingly, patients who received antibiotic therapy did not have a shorter LOS compared to patients treated with supportive therapy and fluids (2.62 days [range 1-56], vs. 2.66 days [range 1-20], $\mathrm{P}=0.77, \mathrm{n}=479$ ). Even when we excluded patients with an extended hospitalization of more than 10 days, the results were similar (2.33 days vs. 2.33, $\mathrm{P}=0.86, \mathrm{n}=467)$. When we looked at patients with presumed sepsis of enteric source, patients who were treated with antibiotic therapy had a slightly shorter LOS, but the difference was not statistically significant ( 2.09 vs. 2.54 days, $\mathrm{P}=0.69$ ).

The empiric use of antibiotic therapy is a common practice not generally supported by strong evidence. Empiric antibiotics are not routinely recommended in the management of AGE except for particular circumstances. The Infectious Diseases Society of America issued new guidelines in 2017 that discouraged the empiric use of antibiotics in immunocompetent adults without signs of severe disease. They recommended limiting antibiotic therapy for patients with presumed sepsis of enteric source ( $\geq 2$ SIRS criteria), immunocompetent patients with documented fever, abdominal pain, bloody diarrhea or bacillary dysentery, or immunocompromised patients with severe illness and bloody diarrhea (strong recommendation but based on a low level of evidence) [16]. Most studies have only shown a small benefit of antibiotic therapy in the clinical course of AGE [17-22]. A meta-analysis from 2012, which included 767 participants from 12 trials, showed no evidence of benefit for antibiotic therapy in non-typhoidal Salmonella diarrheal infection in otherwise healthy people [23]. A second metaanalysis of patients with confirmed Campylobacter species gastroenteritis found that antibiotic treatment shortened the duration of intestinal symptoms by only 1.32 days [24].

Only 15 patients (7.5\%) of our study population had positive stool culture results. The reported yield of stool cultures in previous studies was generally low (1.5-9\%) [25-27]. Our study was conducted prior to the new polymerase chain reaction (PCR)based diagnostic testing techniques that have high sensitivity and a short turnaround time as compared to conventional stool culture testing, with a faster pathogen detection rate [26]. One recent study looked at the use of the gastrointestinal (GI) PCR panel compared to conventional methods such as stool cultures and stool ova and parasite examination [28]. The GI PCR panel group had a higher pathogen detection rate, faster reported results and a lower healthcare cost compared to stool culture. The LOS was shorter by 0.5 days in the GI panel group but the difference was not statistically significant $(\mathrm{P}=0.14)$.

Our findings from this study raise questions as to whether the implementation of empiric antibiotics in cases of acute infectious gastroenteritis is warranted, especially when the existing evidence in the literature is of low quality due to inconsistency and indirectness. The use of antibiotic therapy also comes with risks for medication side effects, promotion of bacterial resistance [29], development of hemolytic uremic syndrome [30], prolonged bacterial stool detection [31], and an increased risk of C. difficile infection.

It was interesting in our study to observe that patients who presented mainly with nausea or vomiting were less likely to receive antibiotics compared to patients with diarrhea or abdominal pain. We believe that this is related to the general consensus that AGE presenting with upper GI symptoms, such as nausea and vomiting, is usually self-limited gastritis that does not require antibiotics. Meanwhile, AGE with lower GI symptoms, such as diarrhea and abdominal pain, is usually more severe and this acute colitis might necessitate antibiotic administration.

Our study included a large number of participants in a very diverse community in New York City and is the first, to our knowledge, to assess the LOS in patients with AGE. There are limitations to our study. It had a retrospective study design; the therapeutic modalities were not randomized and did not take the new GI PCR panel into account. Our study was a singlecenter study with data extracted as coded in electronic medical records. Further, potentially prospective double-blinded, studies are necessary to confirm these findings.

In conclusion, we found no change in the LOS for hospitalized patients with AGE treated with antibiotics compared to supportive therapy, although patients given antibiotics demonstrated more signs and symptoms of the disease. Even in patients with presumed sepsis, the LOS, though slightly shorter, was not statistically significantly different in those who received antibiotic therapy. This calls into question the role of antibiotic therapy in the management of AGE. 


\section{Summary Box}

\section{What is already known:}

- Acute gastroenteritis is a common disease and reason for emergency department visits and hospitalizations

- Most cases of acute gastroenteritis are viral or selflimited bacterial infections

- Antibiotic therapy is only indicated for selected patients with acute gastroenteritis

\section{What the new findings is:}

- Antibiotic therapy had no effect on the length of hospital stay in patients with acute gastroenteritis, even in patients with presumed sepsis

\section{References}

1. Kosek M, Bern C, Guerrant RL. The global burden of diarrhoeal disease, as estimated from studies published between 1992 and 2000. Bull World Health Organ 2003;81:197-204.

2. Roy SL, Scallan E, Beach MJ. The rate of acute gastrointestinal illness in developed countries. J Water Health 2006;4(Suppl 2):31-69.

3. World Health Organization; Foodborne Disease Burden Epidemiology Reference Group. WHO estimates of the global burden of foodborne diseases: 2007-2015. Geneva (CH): WHO; 2015. Available from: https://www.who.int/foodsafety/ publications/foodborne_disease/fergreport/en/[Accessed 25 September 2019].

4. Scallan E, Hoekstra RM, Angulo FJ, et al. Foodborne illness acquired in the United States-major pathogens. Emerg Infect Dis 2011;17:7-15.

5. Levit K, Stranges E, Ryan K EAHF and F. Statistics on hospitalbased care in the United States. Rockville, MD: Agency for healthcare research and quality. 2008. Available from: https://www. hcup-us.ahrq.gov/reports.jsp [Accessed 25 September 2019].

6. Marder EP, Cieslak PR, Cronquist AB, et al. Incidence and trends of infections with pathogens transmitted commonly through food and the effect of increasing use of culture-independent diagnostic tests on surveillance - foodborne diseases active surveillance network, 10 U.S. sites, 2013-2016. MMWR Morb Mortal Wkly Rep 2017;66:397-403. Erratum in: MMWR Morb Mortal Wkly Rep 2017;66:653.

7. Hall AJ, Rosenthal $\mathrm{M}$, Gregoricus $\mathrm{N}$, et al. Incidence of acute gastroenteritis and role of norovirus, Georgia, USA, 2004-2005. Emerg Infect Dis 2011;17:1381-1388.

8. Arena C, Amoros JP, Vaillant V, et al. Acute diarrhea in adults consulting a general practitioner in France during winter: incidence, clinical characteristics, management and risk factors. BMC Infect Dis 2014;14:574.

9. Guerrant RL, Carneiro-Filho BA, Dillingham RA. Cholera, diarrhea, and oral rehydration therapy: triumph and indictment. Clin Infect Dis 2003;37:398-405.

10. Thielman NM, Guerrant RL. Clinical practice. Acute infectious diarrhea. N Engl J Med 2004;350:38-47.

11. Jones R, Rubin G. Acute diarrhoea in adults. BMJ 2009;338:b1877.

12. Levine MM. Antimicrobial therapy for infectious diarrhea. Rev Infect Dis 1986;8(Suppl 2):S207-S216.

13. Vesikari T, Isolauri E, Baer M. A comparative trial of rapid oral and intravenous rehydration in acute diarrhoea. Acta Paediatr Scand 1987;76:300-305.

14. Bellemare S, Klassen TP. Oral versus intravenous rehydration of moderately dehydrated children: a randomized, controlled trial. J Pediatr 2005;147:125-126.

15. Hartling L, Bellemare S, Wiebe N, Russell K, Klassen TP, Craig W. Oral versus intravenous rehydration for treating dehydration due to gastroenteritis in children. Cochrane Database Syst Rev 2006;(3):CD004390.

16. Shane AL, Mody RK, Crump JA, et al. 2017 Infectious diseases society of America Clinical Practice Guidelines for the diagnosis and management of infectious diarrhea. Clin Infect Dis 2017;65:e45-e80.

17. Dryden MS, Gabb RJ, Wright SK. Empirical treatment of severe acute community-acquired gastroenteritis with ciprofloxacin. Clin Infect Dis 1996;22:1019-1025.

18. Goodman LJ, Trenholme GM, Kaplan RL, et al. Empiric antimicrobial therapy of domestically acquired acute diarrhea in urban adults. Arch Intern Med 1990;150:541-546.

19. Zollner-Schwetz I, Krause R. Therapy of acute gastroenteritis: role of antibiotics. Clin Microbiol Infect 2015;21:744-749.

20. Pawlowski SW, Warren CA, Guerrant R. Diagnosis and treatment of acute or persistent diarrhea. Gastroenterology 2009;136:1874-1886.

21. Wiström J, Jertborn M, Ekwall E, et al. Empiric treatment of acute diarrheal disease with norfloxacin. A randomized, placebo-controlled study. Swedish Study Group. Ann Intern Med 1992;117:202-208.

22. DuPont HL. Acute infectious diarrhea in immunocompetent adults. N Engl J Med 2014;370:1532-1540.

23. Onwuezobe IA, Oshun PO, Odigwe CC. Antimicrobials for treating symptomatic non-typhoidal Salmonella infection. Cochrane Database Syst Rev 2012;11:CD001167.

24. Ternhag A, Asikainen T, Giesecke J, Ekdahl K. A meta-analysis on the effects of antibiotic treatment on duration of symptoms caused by infection with Campylobacter species. Clin Infect Dis 2007;44:696-700.

25. Lee JY, Cho SY, Hwang HSH, et al. Diagnostic yield of stool culture and predictive factors for positive culture in patients with diarrheal illness. Medicine (Baltimore) 2017;96:e7641.

26. Wessels E, Rusman LG, van Bussel MJ, Claas EC. Added value of multiplex Luminex Gastrointestinal Pathogen Panel (xTAG ${ }^{\circ}$ GPP) testing in the diagnosis of infectious gastroenteritis. Clin Microbiol Infect 2014;20:O182-O187.

27. Jones TF. How useful are stool studies for acute gastroenteritis? J Infect Dis 2012;205:1334-1335.

28. Beal SG, Tremblay EE, Toffel S, Velez L, Rand KH. A gastrointestinal PCR panel improves clinical management and lowers health care costs. J Clin Microbiol 2018;56.

29. Hakanen A, Jousimies-Somer H, Siitonen A, Huovinen P, Kotilainen P. Fluoroquinolone resistance in Campylobacter jejuni isolates in travelers returning to Finland: association of ciprofloxacin resistance to travel destination. Emerg Infect Dis 2003;9:267-270.

30. Wong CS, Jelacic S, Habeeb RL, Watkins SL, Tarr PI. The risk of the hemolytic-uremic syndrome after antibiotic treatment of Escherichia coli O157:H7 infections. N Engl J Med 2000;342:1930-1936.

31. Sirinavin S, Garner P. Antibiotics for treating salmonella gut infections. Cochrane Database Syst Rev 2000;(2):CD001167. 